\title{
High Speed Area Efficient FFT using Modified SQRT CSLA and 5:3 \& 9:4 Compressor
}

\author{
K.Pitambar Patra ${ }^{1}$, Sambit Patnaik ${ }^{2}$, Swapna Subudhiray ${ }^{3}$, Janmejaya Samal ${ }^{4}$ \\ Asst. Prof., ECE, Einstein Academy of Technology \& Management, Bhubaneswar,India ${ }^{1,2,3,4}$
}

\begin{abstract}
While designing fast fourier transform (FFT) cores, due to the use of multiplexers, memory, or ROMs, there is a substantial increase in power consumption and area. In order to increase speed and throughput, folding and pipelining methods have been approached by various existing designs. But the prime disadvantage of those architectures is the use of multipliers for twiddle multiplications. This present work has proposed fast fourier transform using compressors based multiplier. Both parallel and pipelining techniques have also been used in the proposed designs. Carry Select adder is known to be the fastest adder among the Conventional adder structures. This work uses an efficient Carry select adder by sharing the binary to excess-1 converter (BEC) term. After a logic simplification, we only need one XOR gate, one AND gate and one inverter gate for carry and summation operation. Through the multiplexer, we can select the correct output according to the logic states of the carry in signal. These all design and experiments were carried out on a Xilinx 14.1i Spartan 3e device family.
\end{abstract}

Keywords: Fast Fourier Transform (FFT), Regular 16-bit SQRT CSLA, Modified SQRT CSLA.

\section{INTRODUCTION}

Because of the need of rapid information transmission, today versatile information transfers industry confronts the issue of giving the innovation that have the capacity to bolster a mixed bag of administrations running from voice correspondence with a bit rate of a couple kbps to remote media in which bit rate up to 2 Mbps [1]. The FFT is one of the most commonly used digital signal processing algorithm. Recently, FFT processor has been widely used in digital signal processing field applied for communication systems [2]. FFT/IFFT processors are key components for an Orthogonal Frequency Division Multiplexing (OFDM) based wireless broadband communication system; it is one of the most complex and intensive computation module of various wireless standards. Since FFT is done in the advanced area, there are a few techniques to execute the framework. One of the strategies to actualize the framework is utilizing ASIC (Application Specific Integrated Circuit) [3-4]. ASICs are the quickest, littlest, and least power approach to execute FFT into equipment. The primary issue utilizing this technique is rigidity of configuration procedure included and the more drawn out time to market period for the planned chip [5].

We have proposed outline for executing the quick fourier change (FFT) utilizing modified SQRT CSLA and proposed 5:3 and 9:4 compressors operation in equipment keeping the objective of a force effective structural engineering. These plans are checked utilizing different equipment recreating devices.

\section{5:3 AND 9:4 COMPRESSORS}

To add binary numbers with minimal carry propagation we use compressor adder instead of other adder. Compressor is a digital modern circuit which is used for high speed with minimum gates which requires designing technique. This compressor becomes the essential tool for fast multiplication adding technique by keeping an eye on fast processor and lesser area [6].

\section{- 5:3 Compressor}

5:3 compressors are capable of adding 4 bits and one carry, in turn producing a 3 bit output. The 5-3 compressor has 4 inputs $A_{1}, A_{2}, A_{3}$ and $A_{4}$ and 2 outputs Sum and Carry along with a Carry-in (Cin) and a Carry-out (Cout) as shown in Figure 1. The input Cin is the output from the previous lower significant compressor. .

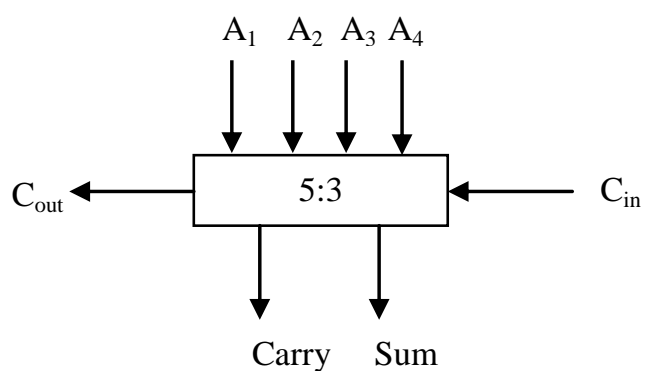

Figure 1: Block Diagram of 4:2 Compressor

The Cout is the output to the compressor in the next significant stage. The critical path is smaller in comparison with an equivalent circuit to add 5 bits using full adders and half adder. The 5-3 compressor is governed by the basic equation

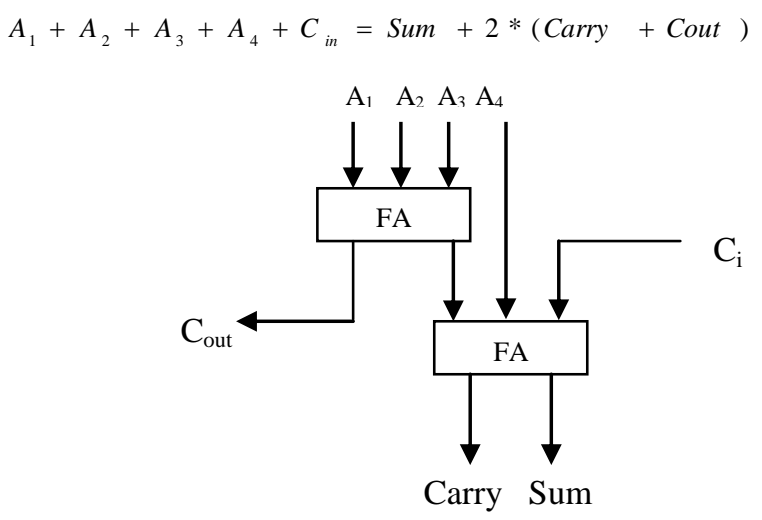

Figure 2(a): Design of 4:2 compressor using Full Adder 
The standard implementation of the 4-2 compressor is done using 2 Full Adder cells as shown in Figure 2(a). When the individual full Adders are broken into their constituent XOR blocks, it can be observed that the overall delay is equal to $4 * \mathrm{XOR}$ [7-8]. The block diagram in Figure 2(b) shows the existing architecture for the implementation of the 4-2 compressor with a delay of $3 *$ XOR. The equations governing the outputs in the existing architecture are shown below

$$
\begin{aligned}
\text { Sum } & =A_{1} \oplus A_{2} \oplus A_{3} \oplus A_{4} \oplus C_{i n} \\
\text { Cout } & =\left(A_{1} \oplus A_{2}\right) \cdot A_{3}+\overline{\left(A_{1} \oplus A_{2}\right)} \cdot A_{1}
\end{aligned}
$$

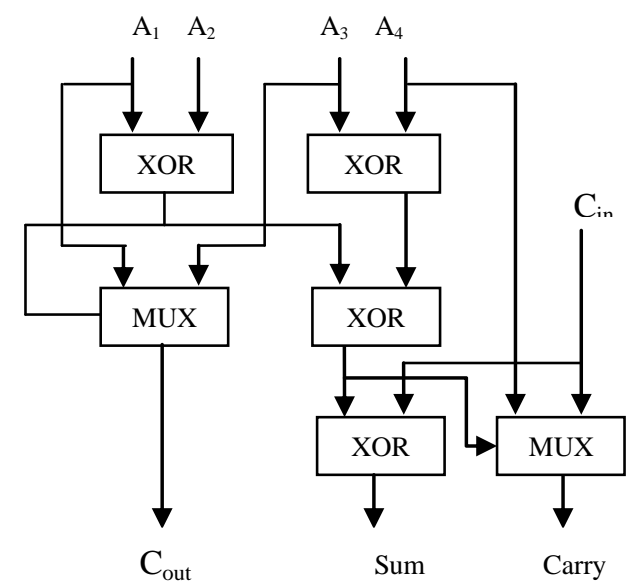

Figure 2(b): 4:2 Compressor using XOR and Multiplexer

$$
\text { Carry }=\frac{\left(A_{1} \oplus A_{2} \oplus A_{3} \oplus A_{4}\right) \cdot C_{i n}+}{\left(A_{1} \oplus A_{2} \oplus A_{3} \oplus A_{4}\right) \cdot A_{4}}
$$

Thus replacing some XOR blocks with multiplexer's results in a significant improvement in delay. Also the MUX block at the SUM output gets the select bit before the inputs arrive and thus the transistors are already switched by the time they arrive. This minimizes the delay to a considerable extent. This is shown in Figure 2(c).

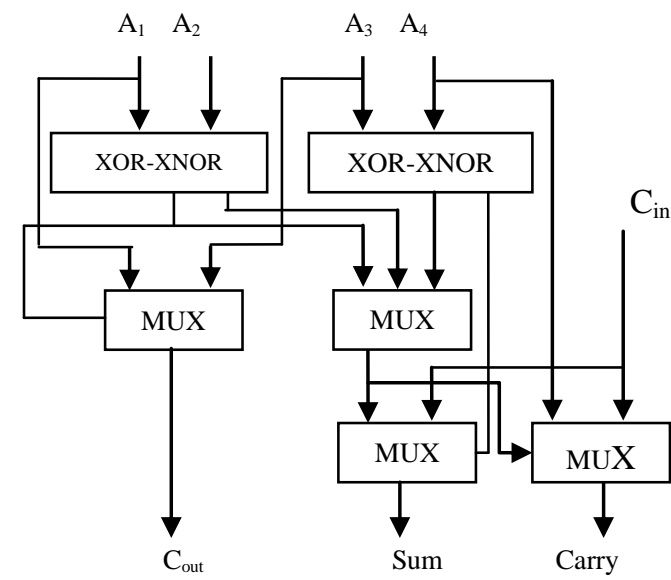

Figure 2(c): Proposed 4:2 Compressor using XOR-XNOR Gate

The equations governing the outputs in the proposed architecture are shown below

$$
\text { Sum }=\left(A_{1} \oplus A_{2}\right) \cdot\left(A_{3} \oplus A_{1}\right)+\left(A_{1} \oplus A_{2}\right) \cdot C_{\text {in }}
$$

$$
\begin{aligned}
& \text { Cout }=\left(A_{1} \oplus A_{2}\right) \cdot A_{3}+\overline{\left(A_{1} \oplus A_{2}\right)} \cdot A_{1} \\
& \text { Carry }=\left(A_{1} \oplus A_{2} \oplus A_{3} \oplus A_{4}\right) \cdot C_{\text {in }}+ \\
& \overline{\left(A_{1} \oplus A_{2} \oplus A_{3} \oplus A_{4}\right)} . A_{4}
\end{aligned}
$$

\section{- 9:4 Compressor}

Similar to its 5:3 compressor counterpart, the 9:4 compressors as shown in Figure 3, is capable of adding 7 bits of input and 2 carry's from the previous stages, at a time [9]. In our implementation, we have designed a novel 9:4 compressor utilizing two 5:3 compressors, two full adder and one half adders.

The architecture for the same has been shown in Figure 4.

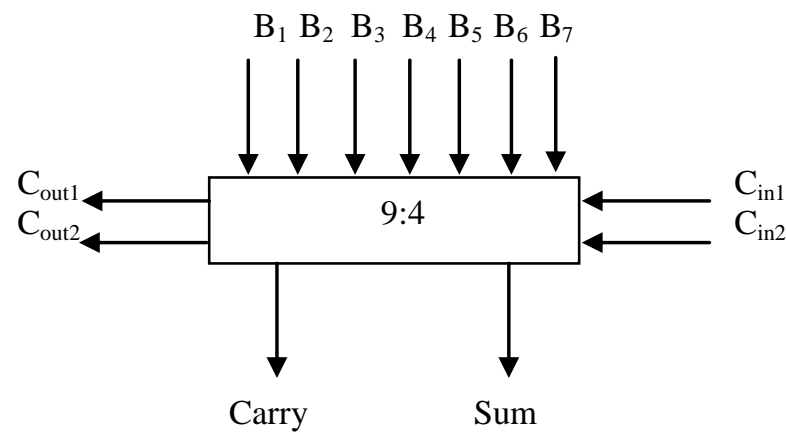

Figure 3: Block Diagram of 7:2 Compressors

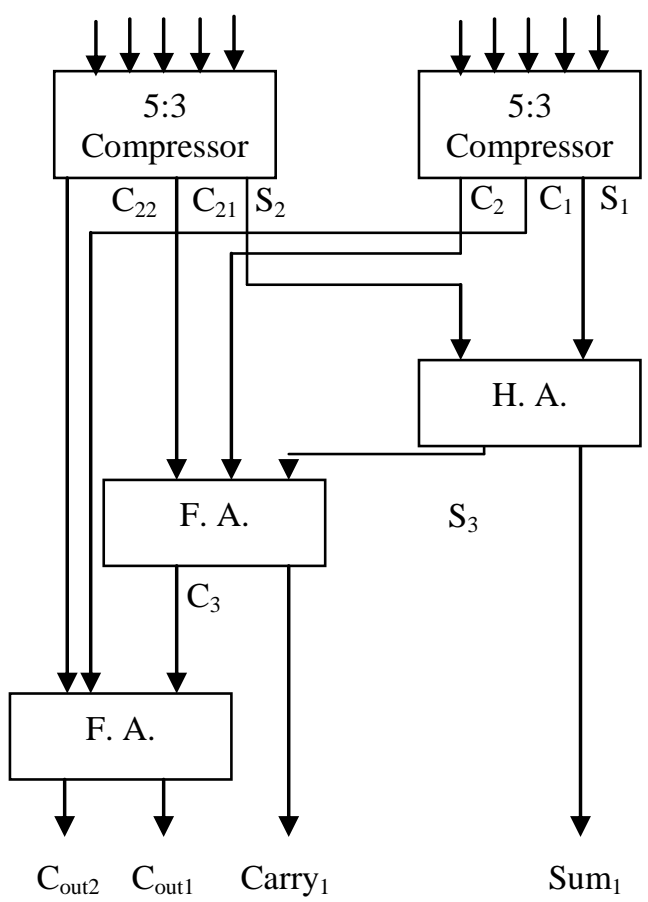

Figure 4: 9:4 Compressor using 5:3 Compressor

$$
\begin{aligned}
& \text { Sum } 1=S_{1} \oplus S_{2} \\
& \text { Carry } 1=S_{3} \oplus C_{1} \oplus C_{21} \\
& C_{\text {out } 1}=C_{3} \oplus C_{2} \oplus C_{22} \\
& C_{\text {out } 2}=C_{3} C_{2}+C_{22} C_{2}+C_{3} C_{22}
\end{aligned}
$$



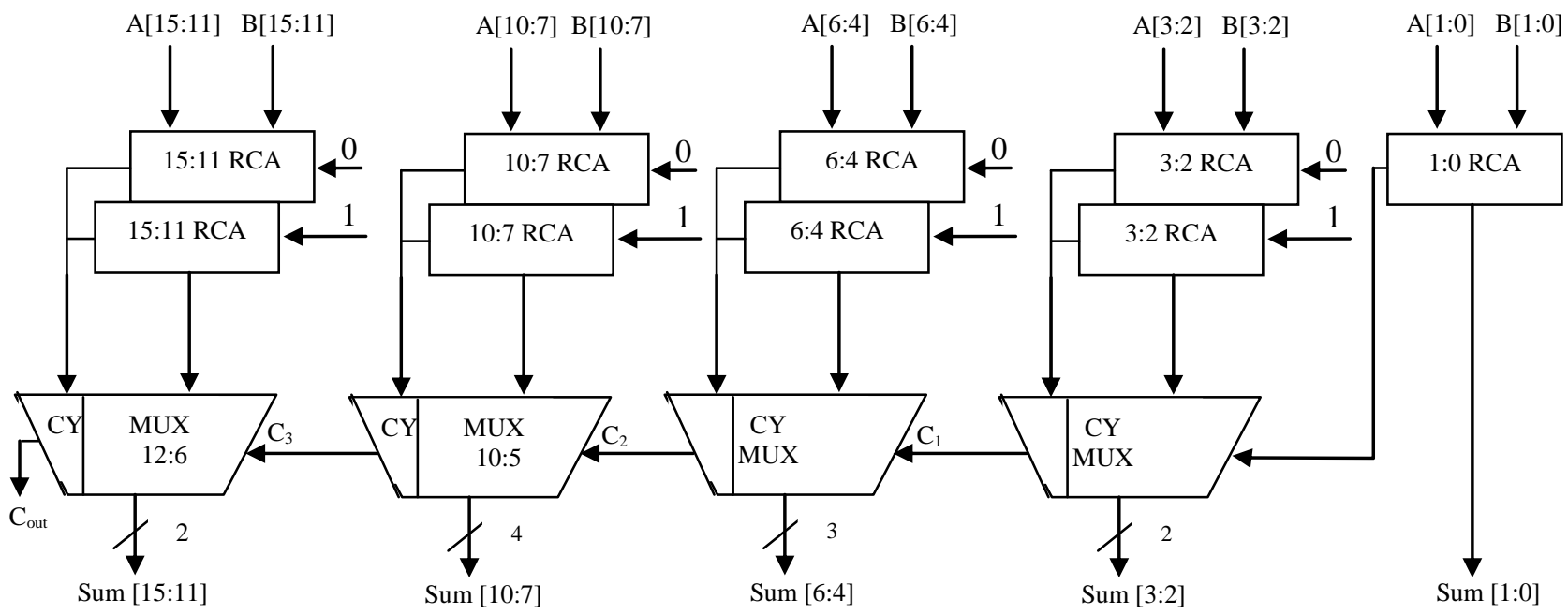

Figure 5: Block Diagram of 16-bit Regular 16-bit SQRT CSLA using RCA

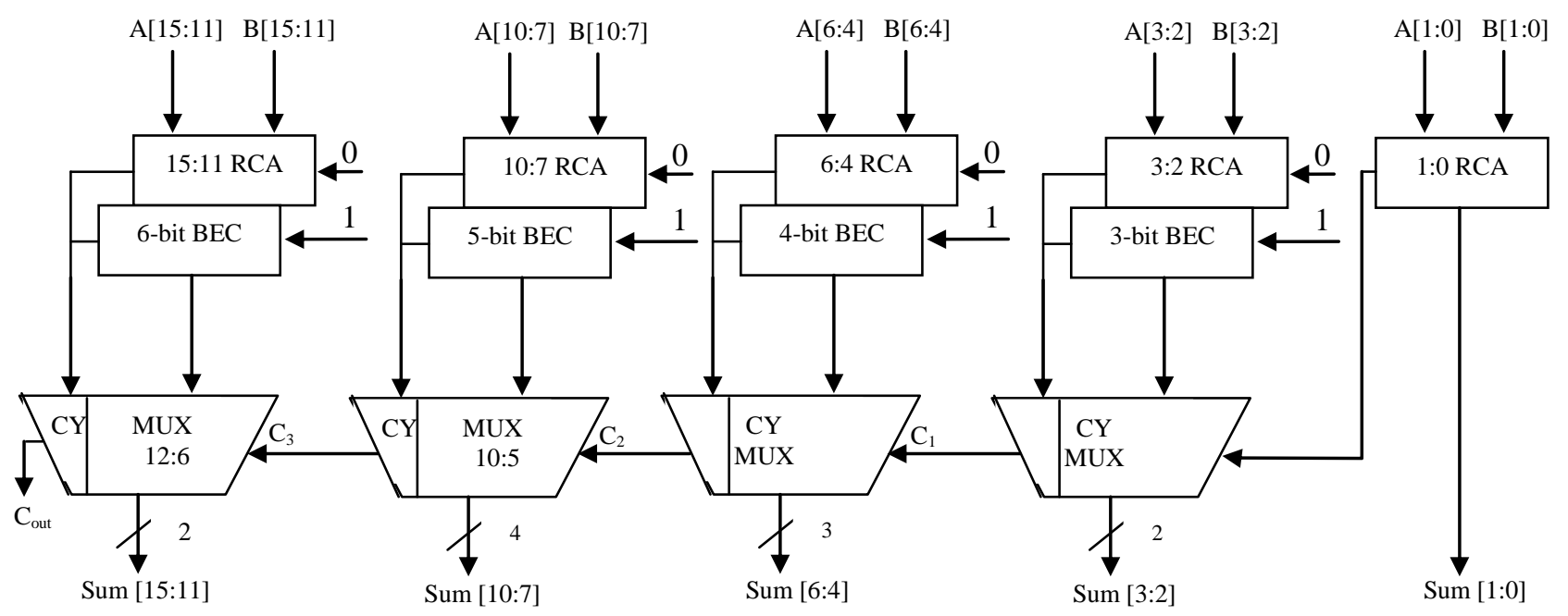

Figure 6: Block Diagram of Regular 16-bit SQRT CSLA using RCA with BEC

\section{REGULAR 16-BIT SQRT CSLA USING RCA}

The regular 16-bit SQRT CSLA is a comprises of two swell convey adders and a multiplexer. Including two n-bit numbers with a convey select viper is finished with two adders (in this way two swell convey adders) keeping in mind the end goal to perform the computation twice, one time with the presumption of the convey being zero and the other accepting one. After the two outcomes are ascertained, the right aggregate, and the right convey, is then chosen with the multiplexer once the right convey is known [10].

A 16-bit convey select snake can be created in two unique sizes to be specific uniform piece size and variable square size. Swell convey adders are the easiest and smaller full adders, however their execution is restricted by a convey that must proliferate from the minimum noteworthy bit to the most-critical bit. The pace of a convey select viper can be enhanced upto $40 \%$ to $90 \%$, by performing the increases in parallel, and diminishing the most extreme convey delay. Figure 5 demonstrates the Regular structure of 16-bit SQRT CSLA.
It incorporates numerous swell convey adders of variable sizes which are separated into gatherings. Bunch 0 contains 2-bit RCA which contains stand out swell convey snake which includes the info bits and the information convey and results to entirety [1:0] and the do. The do of the Group 0 which goes about as the choice data to mux which is in gathering 1 , chooses the outcome from the relating RCA $(\mathrm{Cin}=0)$ or RCA $(\mathrm{Cin}=1)$. Additionally the remaining gatherings will be chosen relying upon the Cout from the past gatherings.

\section{- MODIFIED SQRT CSLA}

The Binary to excess one Converter (BEC) replaces the ripple carry adder with $\mathrm{Cin}=1$, in order to reduce the area and power consumption of the regular CSLA. The modified16-bit CSLA using BEC is shown in Figure 6. The structure is again divided into five groups with different bit size RCA and BEC. The group 2 of the modified 16-bit CSLA is shown Figure 6

- BINARY TO EXCESS-1 CONVERTER (BEC)

BEC is a technique to reduce the number of partial products in 1-bit increase. The binary to excess-1 
converter is used to reduce the area and power consumption in CSA. Figure 7 shows the basic structure of 3-b BEC. The Boolean expressions of the 3-b BEC is as

$$
\begin{aligned}
\mathrm{X} 0 & =\sim \mathrm{B} 0 \\
\mathrm{X} 1 & =\mathrm{B} 0^{\wedge} \mathrm{B} 1 \\
\mathrm{X} 2 & =\mathrm{B} 2^{\wedge}(\mathrm{B} 0 \& \mathrm{~B} 1 \& \mathrm{~B} 2)
\end{aligned}
$$

Table 1: Function Table of 3-bit BEC

\begin{tabular}{|c|c|}
\hline Binary[2:0] & Excess- 1[2:0] \\
\hline 000 & 001 \\
001 & 010 \\
010 & 011 \\
$\mid$ & $\mid$ \\
$\mid$ & $\mid$ \\
111 & 000 \\
\hline
\end{tabular}

By manually counting the number of gates used for group 2 is 43 (full adder, half adder, multiplexer, BEC). One input to the mux goes from the RCA with Cin=0 and other input from the BEC. Comparing the group 2 of both regular and modified CSLA, it is clear that BEC structure reduces the area and power. But the disadvantage of BEC method is that the delay is increasing than the regular CSLA. The main idea of binary to exces-1 converter instead of the RCA with RCA (ripple carry adder) and Cin $=1$ in order to reduce the area and power consumption of the 16-B SQRT CSA.

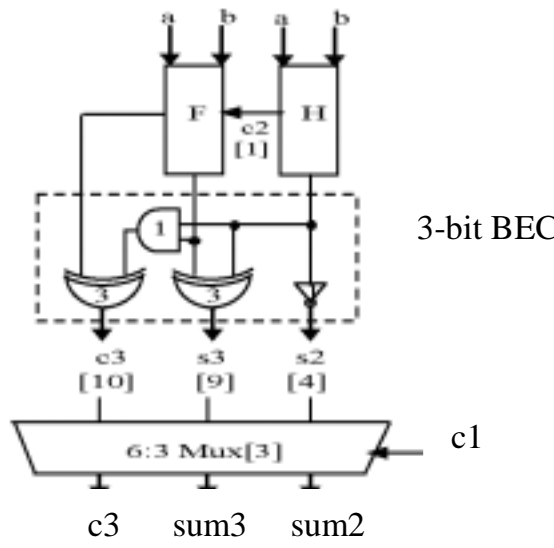

Figure 7-bit Booth Encoder

\section{- DELAY AND AREA METHODOLOGY}

The delay and area evaluation methodology considers all gates to be made up of AND, OR, and Inverter (AOI), each having delay equal to 1 unit and area equal to 1 unit. We then add up the number of gates in the longest path of a logic block that contributes to the maximum delay. The area evaluation is done by counting the total number of AOI gates required for each logic block [11-12].

Table 2: Delay and Area count of the basic block of CSLA

\begin{tabular}{|c|c|c|}
\hline Adder Block & Delay & Area \\
\hline XOR & 3 & 5 \\
\hline 2:1 MUX & 3 & 4 \\
\hline Half Adder & 3 & 6 \\
\hline Full Adder & 6 & 13 \\
\hline
\end{tabular}

Table 3: Delay and Area Count of Regular SQRT CSLA and Modified SQRT CSLA Groups

\begin{tabular}{|c|c|c|c|c|c|}
\hline \multicolumn{2}{|c|}{ Regular SQRT CSLA } & \multicolumn{3}{c|}{ Modified SQRT CSLA } \\
\hline Group & Delay & Area & Group & Delay & Area \\
\hline Group-1 & 9 & 19 & Group-1 & 9 & 19 \\
\hline Group-2 & 11 & 57 & Group-2 & 13 & 43 \\
\hline Group-3 & 13 & 87 & Group-3 & 16 & 61 \\
\hline Group-4 & 16 & 117 & Group-4 & 19 & 84 \\
\hline Group-5 & 19 & 147 & Group-5 & 22 & 107 \\
\hline
\end{tabular}

\section{FAST FOURIER TRANSFORM}

The decimation, however, causes shuffling in data. The entire process involves $v=\log 2 N$ stages of decimation. Fast Fourier Transform (FFT) is one of the most efficient ways to implement Discrete Fourier Transform (DFT) due to its reduced usage of arithmetic units. DFT is one of those primary tools that are used for the frequency analysis of discrete time signals and to represent a discrete time sequence in frequency domain using its spectrum samples. The analysis (forward) and synthesis (inverse) equations of an $\mathrm{N}$ point FFT are given below.

$X[k]=\sum_{n=0}^{N-1} x[n] W_{N}^{k n}$
$x[n]=\frac{1}{N} \sum_{n=0}^{N-1} X[k] W_{N}^{-k n}$

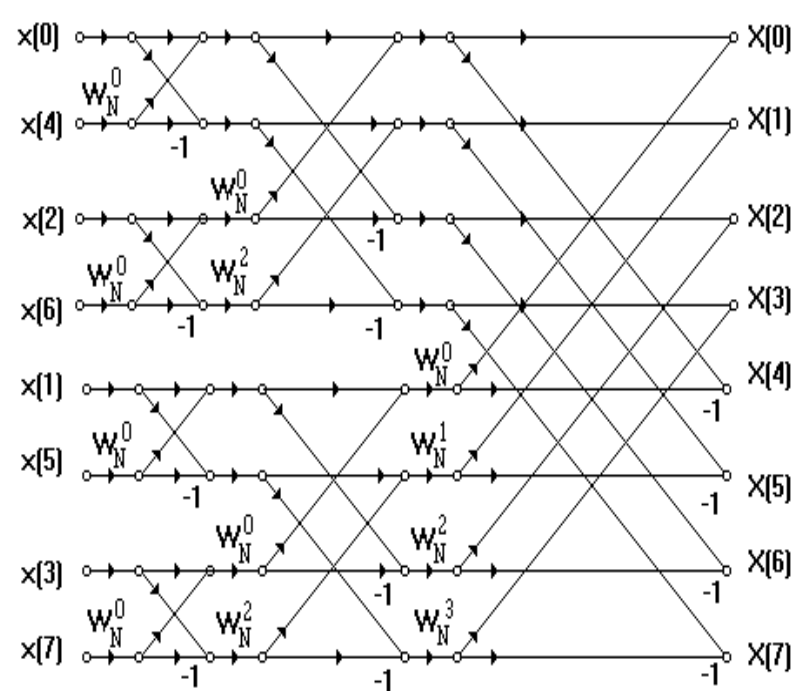

Figure 8: IFFT Signal Flow Graph.

Graphically the operation can be view using FFT flow graph shown in figure 8 . From this figure, the FFT computation is accomplished in three stages. The $\mathrm{X}(0)$ until X (7) variable is denoted as the input value divided as even and odd terms for FFT computation and X (0) until X (7) is comes in a sequence form as the output. There are two operations to complete the computation in each stage. The upward arrow will execute addition operation while downward arrow will execute subtraction operation. The subtracted value is multiplied with twiddle factor value before being processed into the next stage. This operation is done concurrently and is known as butterfly process. Noted that each of the butterfly process is performed 
concurrently enable it to execute FFT computation process in a very fast technique.

\section{SIMULATION RESULT}

All the designing and experiment regarding algorithm that we have mentioned in this paper is being developed on Xilinx 14.1i updated version. Xilinx 14.1i has couple of the striking features such as low memory requirement, fast debugging, and low cost. The latest release of ISE ${ }^{\mathrm{TM}}$ (Integrated Software Environment) design tool provides the low memory requirement approximate 27 percentage low. By the aid of that software we debug the program easily. Also included is the newest release of the chip scope Pro Serial IO Tool kit, providing simplified debugging of high-speed serial IO designs for Spratan-3 FPGAs. We functionally verified each unit presented in this paper including modified SQRT CSLA and proposed compressor based multiplier. We have been found from the results shown in Table 2 to Table 4 respectively.

Table 4: Device utilization summary (VERTEX-7) of Fast Fourier Transform (FFT)

\begin{tabular}{|l|c|c|c|}
\hline \multicolumn{1}{|c|}{ Design } & $\begin{array}{c}\text { No. of } \\
\text { slices }\end{array}$ & $\begin{array}{c}\text { No. of 4 } \\
\text { input LUTs }\end{array}$ & $\begin{array}{c}\text { MCPD } \\
\text { (ns) }\end{array}$ \\
\hline $\begin{array}{l}\text { FFT using Modified } \\
\text { SQRT CSA and } \\
\text { Compressor based } \\
\text { Multiplier }\end{array}$ & 327 & 576 & 22.269 \\
\hline $\begin{array}{l}\text { FFT using Modified } \\
\text { SQRT CSA and } \\
\text { Compressor based } \\
\text { Multiplier }\end{array}$ & 171 & 302 & 16.369 \\
\hline $\begin{array}{l}\text { FFT using Modified } \\
\text { SQRT CSA and } \\
\text { Proposed Compressor } \\
\text { based Multiplier }\end{array}$ & 149 & 261 & 13.965 \\
\hline
\end{tabular}

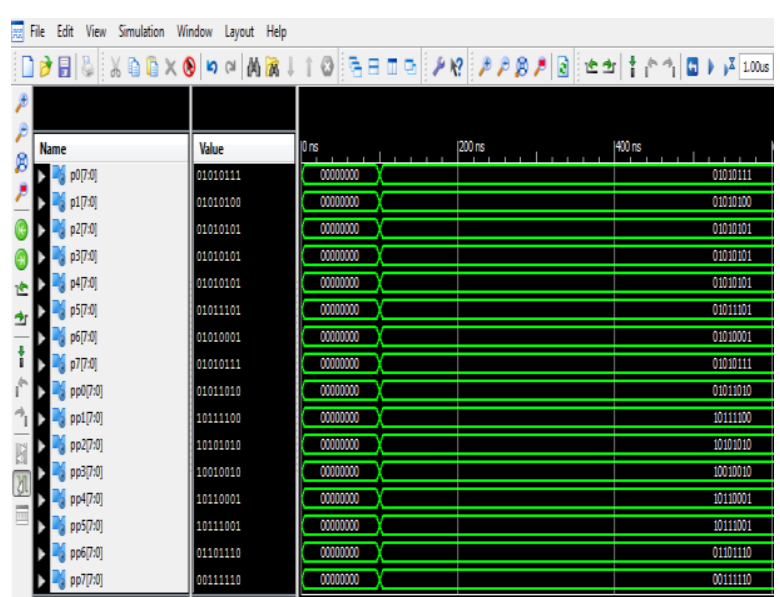

Figure 9: Output Waveform of Proposed Design

\section{CONCLUSION}

While designing various FFT cores, due to the use of multiplexers, memory, or ROMs, there is a substantial increase in power consumption and area. In order to increase speed and throughput, folding and pipelining methods have been approached by various existing designs. But the prime disadvantage of those architectures is the use of multipliers for twiddle multiplications. This present work has proposed FFT using compressors based multiplier. Both parallel and pipelining techniques have also been used in the proposed designs. A compressor adder is a logical circuit which is used to improve the computational speed of the addition of 4 or more bits at a time. The proposed design is hardware efficient as compared to other traditional methods as well as architectures that are built using only compressors.

\section{REFERENCES}

11] Sushma R. Huddar and Sudhir Rao, Kalpana M., "Novel High Speed Vedic Mathematics Multiplier using Compressors", 978-14673-5090-7/13/\$31.00 C2013 IEEE.

[2] S. S. Kerur, Prakash Narchi, Jayashree C N, Harish M Kittur and Girish V A, "Implementation of Vedic multiplier for Digital Signal Processing", International Conference on VLSI, Communication \& Instrumentation (ICVCI) 2011, Proceedings published by International Joural of Computer Applications ${ }^{\circledR}$ (IJCA), pp.1-6.

[3] Himanshu Thapaliyal and M.B Srinivas, "VLSI Implementation of RSA Encryption System Using Ancient Indian Vedic Mathematics", Center for VLSI and Embedded System Technologies, International Institute of Information Technology Hyderabad, India.

[4] Jagadguru Swami Sri Bharati Krishna Tirthaji Maharaja, "Vedic Mathematics: Sixteen simple Mathematical Formulae from the Veda", Delhi(2011)

[5] Sumit Vaidya and Depak Dandekar. "Delay-power perfor-mance comparison of multipliers in VLSI circuit design". International Journal of Computer Networks \& Communications (IJCNC), Vol.2, No.4, July 2010.

[6] P. D. Chidgupkar and M. T. Karad, "The Implementation of Vedic Algorithms in Digital Signal Procesing", Global J. of Eng. Edu, Vol.8, No.2, 204, UICEE Published in Australia.

[7] S. Correa, L. C. Freitas, A. Klautau and J. C. W. A. Costa, "VHDL Implementation of a Flexible and Synthesizable FFT Processor", IEEE LATIN AMERICA TRANSACTIONS, VOL. 10, NO. 1, JAN. 2012

[8] Y. Kim and L. -S. Kim, "16-bit carry-select adder with reduced area,"Electron. Lett. vol. 37, no. 10, pp. 614-615, May 2001.

[9] B. Ramkumar, H. M. Kittur, and P. M. Kannan, "ASIC implementation of modified faster carry save adder," Eur. J. Sci. Res., vol. 42, no. 1, pp. 53-58, 2010.

[10] T. Y. Ceiang and M. J. Hsiao, "Carry-select adder using single ripple carry adder," Electron. Lett., vol. 34, no. 22, pp. 2101-2103, Oct. 1998.

[11] Y. Kim and L.-S. Kim, "64-bit carry-select adder with reduced area," Electron. Lett., vol. 37, no. 10, pp. 614-615, May 2001.

[12] J. M. Rabaey, Digtal Integrated Circuits-A Design Perspective. Upper Saddle River, NJ: Prentice-Hall, 2001.

[13] Y. He, C. H. Chang, and J. Gu, "An area efficient 64-bit square root carry-select adderfor low power applications,"in Proc.IEEE Int. Symp. Circuits Syst., 2005, vol. 4, pp. 4082-4085. 